Canadian

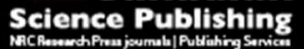

Canadian Journal of Civil Engineering Revue canadienne de génie civil

\title{
GESTION DES DÉBITS D'UN BARRAGE EN MILIEU URBANISÉ
}

\begin{tabular}{|r|l|}
\hline Journal: & Canadian Journal of Civil Engineering \\
\hline Manuscript ID & cjce-2016-0241.R1 \\
\hline Manuscript Type: & Article \\
\hline Date Submitted by the Author: & 30 -Aug-2016 \\
\hline Complete List of Authors: & $\begin{array}{l}\text { Roy-Gosselin, Philippe-Hubert; Universite Laval, Génie civil et génie des } \\
\text { eaux } \\
\text { Chouinard, Bastien; Ville de Quebec, Qualité du milieu - Service de } \\
\text { l'environnement } \\
\text { Morse, Brian; Universite Laval, Génie civil et génie des eaux } \\
\text { Pelletier, Geneviève; Université Laval, Département de génie civil et de } \\
\text { génie des eaux }\end{array}$ \\
\hline Keyword: & $\begin{array}{l}\text { environmental < MANUSCRIPT CLASSIFICATION, french only < type of } \\
\text { paper to review, erosion and sediment < Hydrotechnical Eng., rivers-lakes- } \\
\text { est-\& reserv < Hydrotechnical Eng., barrage }\end{array}$ \\
\hline \multicolumn{2}{|c}{} \\
\hline
\end{tabular}




\section{GESTION DES DÉBITS D'UN BARRAGE EN MILIEU URBANISÉ}

Philippe-Hubert Roy-Gosselin, Ingénieur, M. Sc., Diplômé du Département de génie civil et génie des eaux, Université Laval, Pavillon Adrien-Pouliot, 1065, avenue de la Médecine, Québec (Québec) Canada G1V 0A6,

Philippe-Hubert.Roy-Gosselin@mtq.gouv.qc.ca

Bastien Chouinard, Technicien, Qualité du milieu - Service de l'environnement, Ville de Québec, 250L, boulevard Wilfrid-Hamel (Pavillon des arts), Québec, (Québec) G1L 5A7, bastien.chouinard@,ville.quebec.qc.ca

Brian Morse, Professeur, Département de génie civil et génie des eaux, Université Laval, Pavillon Adrien-Pouliot, 1065, avenue de la Médecine, Québec (Québec) Canada G1V 0A6, Brian.Morse@gci.ulaval.ca

*Geneviève Pelletier, Professeure, Département de génie civil et génie des eaux, Université Laval, Pavillon Adrien-Pouliot, 1065, avenue de la Médecine, Québec (Québec) Canada G1V 0A6, Genevieve.Pelletier@gci.ulaval.ca; 418-656-2647

*Auteur correspondant

Compte de mots : 6029 


\section{RÉSUMÉ}

Le barrage Joseph-Samson est situé à l'embouchure de la rivière Saint-Charles au confluent du fleuve Saint-Laurent dans la Basse-Ville de Québec. Il crée un plan d'eau sur environ 3,7 km à l'amont du barrage, bordé d'un parc linéaire très fréquenté par les citoyens. Le plan d'eau a une valeur esthétique et permet la navigation de loisir. Avec ses clapets anti-marées, le barrage protège la Basse-Ville lorsqu'il y a de très grandes marées vives dans le fleuve Saint-Laurent. Par contre, le barrage a complètement changé un estuaire naturel et vivant en canal de sédimentation de matière organique et inorganique provenant du bassin versant forestier, semi-urbain et urbain. Le libre passage des poissons, qui étaient autrefois abondants, est entravé. De plus, une réduction importante d'oxygène dissous est souvent observée. Le barrage pourrait même contribuer aux risques d'inondation lorsque le débit dans la Saint-Charles est élevé. Cet article présente les résultats des simulations 1D (HEC-RAS) pour évaluer l'impact de la présence du barrage sur les risques d'inondation pour différents scénarios d'ouverture et fermeture de sa vanne de fond. De plus, il présente les résultats des simulations 2D (River2D) pour évaluer l'impact du barrage sur les vitesses et les processus de sédimentation. 


\section{INTRODUCTION}

Par ses clapets anti-marées, le barrage Samson sur la rivière Saint-Charles à Québec a bien rempli sa mission de protection contre les inondations jusqu'à maintenant. Par contre, il a aussi créé un plan d'eau sur quelques kilomètres en amont, créant une plus grande aire d'écoulement, une vitesse moins élevée et des conditions idéales pour le dépôt de sédiments. Hydrauliquement, la sédimentation et le rehaussement des niveaux d'eau réduisent la capacité d'évacuation des réseaux pluviaux. Une baisse de cette capacité viendrait augmenter les risques d'inondations par les égouts pluviaux drainant la Basse-Ville de Québec. Ce problème de sédimentation et d'obstruction des conduites doit donc être bien surveillé. Au niveau de la faune, la sédimentation contribue à la destruction des écosystèmes (Yin 2010) et à l'envasement des frayères. Le Service de l'eau et de l'environnement de la Ville de Québec a d'ailleurs déjà recensé des espèces qui ont disparu de la rivière, dont les exemples les plus frappant est la perte de frayère de l'éperlan et la disparition de l'esturgeon jaune (Société de la rivière Saint-Charles 2007). Le plan d'eau artificiel créé par le barrage réduit la vitalité et la diversité des écosystèmes par la baisse de l'oxygène dissous due au long temps de séjour de l'eau (Compagnon 2000) et en limitant la variation des niveaux d'eau et le brassage des eaux, deux facteurs importants pour les écosystèmes (Yin 2010). À cela s'ajoute la liaison hydraulique avec le fleuve Saint-Laurent qui se trouve coupée, ce qui empêche les poissons de circuler librement et de remonter pour le frai.

Quelques études ont été réalisées sur l'impact hydrologique des barrages au Québec, dont Assani et al. (2002) qui ont analysé ces impacts sur le régime hydrologique de la rivière Matawin. Ils ont montré que les barrages réduisent la proportion de l'écoulement annuel transitée au printemps et laminent les débits maximaux mensuels. Assani et al. (2005) ont étudié l'effet des barrages sur les débits d'étiage en comparant les données de 72 stations hydrométriques. Les barrages 
diminuent en général les débits annuels minimaux. Ces changements hydrologiques ont un impact sur la morphologie des cours d'eau. Alibert et al. (2011) ont effectué une étude portant sur les impacts morphologiques à l'amont d'un barrage au Québec sur la rivière Matawin, démontrant que les changements morphologiques attendus sont reliés à la quantité de sédiments charriés par la rivière en amont du barrage.

Avec l'urbanisation de la région de Québec, la charge sédimentaire charriée par la rivière SaintCharles a augmenté alors que celle exportée au fleuve Saint-Laurent a diminué suite à la construction du barrage Samson. Tel que rapporté par Syvitsky et al. (2015), ceci est un phénomène global. Ils estiment que les humains ont augmenté la charge sédimentaire des rivières de 2,3 milliards tonnes tout en réduisant celle sortant des estuaires de 1,4 milliards tonnes. La littérature présente bien le phénomène de retenue des sédiments par les barrages et l'impact morphologique de ces retenues sur les rivières. Cependant, l'utilisation du phénomène des marées pour rétablir un milieu ayant subi d'importants changements morphologiques suite à la construction d'un barrage reste un sujet peu étudié. Les connaissances sur les impacts d'un barrage anti-marée sur l'écoulement en zone urbaine sont encore limitées au Québec.

Le dépôt des sédiments est une problématique en milieu aquatique agricole et urbain, néanmoins le transport de sédiments est un phénomène bien documenté sur lequel il y a de nombreuses études. La littérature présente de nombreux modèles d'érosion, de transport et de dépôt des sédiments (Lick 2009). Le cas réel de la rivière Petitcodiac, au Nouveau-Brunswick, a démontré hors de tout doute que le pont-chaussée et les clapets anti-marées causent, au fil des années, une accumulation massive de sédiments près du barrage. De plus, la faune aquatique, composée de poissons migrateurs, avait pratiquement disparu de cette rivière à cause des mauvaises conditions 
causées par les sédiments et surtout à cause du lien hydraulique coupé par le barrage avec le golfe du Saint-Laurent. Les Sentinelles de la Petitcodiac est le groupe responsable de l'ouverture des vannes et du suivi de la qualité de l'écosystème (Sentinelles de la rivière Petitcodiac 2014). Les vannes du barrage ont été ouvertes en 2010 sous la pression du public et des organismes locaux. L'année même de l'ouverture, les sédiments ont commencé à être évacués et la faune aquatique a amorcé son retour. De nombreux changements positifs ont été répertoriés lors des deux premières années. Le passage du poisson s'est nettement amélioré, les risques d'inondation dans la région ont diminué et la reprise d'un fort mascaret à l'aval du barrage a été observée. En effet, la rivière à l'aval s'est considérablement élargie et des espèces de poissons autrefois absentes sont revenues (Sentinelles de la rivière Petitcodiac 2013).

Similairement, des observations sur la partie aval de la rivière Saint-Charles ont permis de constater l'étendue des effets de la sédimentation en amont du barrage. Cette étude présente des simulations effectuées à l'aide des modèles HEC-RAS et River2D dans différentes conditions de débits et de marées afin d'évaluer les avantages d'ouvrir la vanne de fond du barrage lors des cycles de marnage normaux, tant au niveau de la qualité des écosystèmes qu'au niveau de l'évacuation des sédiments. L'hypothèse de travail est la suivante : l'ouverture de la vanne offre des vitesses d'écoulement assez bonnes pour l'évacuation du limon accumulé en amont du barrage et permet un échange important avec les eaux mieux oxygénées du fleuve Saint-Laurent.

\section{SECTEUR À L'ÉTUDE}

\section{Site}

La rivière Saint-Charles, qui collecte les eaux d'un territoire de $550 \mathrm{~km}^{2}$, subit l'influence des puissantes marées (marnage de $6 \mathrm{~m}$ ) du fleuve Saint-Laurent. Le barrage-poids en béton Joseph- 
Samson a été mis en eau en 1970 pour atténuer des risques d'inondation causés par les hautes marées et créer un plan d'eau submergeant les émissaires des égouts unitaires et pluviaux dans la rivière. Ces marées pénètrent dans la rivière Saint-Charles jusqu'à la hauteur du pont Marie-deL'Incarnation, à 3,7 km à l'amont du barrage (Figure 1). Le barrage est situé sous un pont ferroviaire à côté du boulevard Jean-Lesage.

Le site à l'étude est la partie aval de la rivière Saint-Charles, entre le barrage Samson et le pont Marie-de-L'Incarnation. Le tronçon est présenté à la Figure 1. Ce site est situé dans la BasseVille de Québec et le tronçon de rivière à l'étude fait un peu plus de $3 \mathrm{~km}$. Ce choix de zone à l'étude découle d'une étude réalisée par le Service de l'environnement de la Ville de Québec pour évaluer les zones qui seraient affectées par les marées si le barrage Samson était retiré. De plus, ce sont des secteurs affectés par la sédimentation. Les zones vertes et brunes sur cette figure représentent des lieux propices à la formation de milieux humides si le barrage n'était pas présent (Ville de Québec 2012).

Par ailleurs, les rues dans les quartiers environnants sont situées généralement entre une élévation géodésique de 7 et $8 \mathrm{~m}$ et des digues sont aménagées le long la rivière. Si le niveau des eaux de la rivière devait monter à plus de la cote de $5 \mathrm{~m}$, il y aurait risque de refoulement dans les égouts situés à $2 \mathrm{~m}$ sous la surface de la rue. Les quartiers autour de la rivière étant vieux, il est possible que les clapets anti-refoulement soient vétustes et qu'ils ne protègent pas adéquatement les soussols des bâtiments. C'est pourquoi une élévation de la surface de l'eau dépassant $5 \mathrm{~m}$ dans la rivière serait considérée comme à risque pour la perte d'efficacité d'évacuation des égouts unitaires et pluviaux. Vingt-quatre émissaires se jettent dans ce tronçon de rivière. De plus, la Ville de Québec a mis en place un réseau de bassins de rétention des eaux unitaires vers lesquels 
sont redirigés bon nombre de collecteurs. Les trop-pleins de ces bassins déversent encore leurs eaux dans la rivière Saint-Charles.

\section{Barrage}

La désignation du barrage au MDDELCC (anciennement le Centre d'Expertise Hydrique du Québec, CEHQ) est « X0007700 ». Il est doté de déversoirs munis de clapets anti-marées (installés en 1974) et d'une vanne de fond opérée manuellement. Le pont a six piliers dans le cours d'eau et la vanne de fond est située entre les piliers 3 et 4 . La vanne de fond permet la vidange des eaux de la rivière. Faite en acier, elle a une longueur de $9725 \mathrm{~mm}$, une épaisseur de $762 \mathrm{~mm}$ et une hauteur de $2743 \mathrm{~mm}$. La vanne possède un accès par le dessus pour l'entretien. Le seuil sur lequel repose la vanne se situe à une élévation géodésique de 0,6 $\mathrm{m}$. La vanne est généralement fermée. L'ouverture se fait à partir d'un petit chemin d'accès sous le pont qui devient difficilement accessible lors de fortes crues.

Les six pertuis totalisant 32 clapets anti-marées, dont les radiers sont à une élévation géodésique de 3,05 m, sont d'une hauteur de 2,2 mètres chacun et couvre une largeur totale de $85 \mathrm{~m}$. Ces clapets permettent d'évacuer les eaux de la rivière vers le fleuve, mais empêchent les eaux du fleuve d'entrer lorsque la marée surpasse l'élévation maximale du barrage et de la rivière. De tels événements de fortes marées se produisent chaque année pendant les marées de vives eaux observées au printemps et à l'automne.

\section{MÉTHODOLOGIE}

Le projet comporte deux phases dans sa réalisation. La phase la plus importante du projet est la modélisation du tronçon à l'étude au moyen des logiciels HEC-RAS (U.S. Army Corps of 
Engineers 2010) et River2D (Steffler et Blackburn 2002) et la simulation de différents scénarios de conditions hydrauliques pour étudier le comportement de la rivière en termes de niveaux d'eaux maximaux et minimaux, de vitesses maximales et de risques d'inondation. Une inondation mineure (niveau d'eau au-dessus de la cote de $5 \mathrm{~m}$ ) provoque un refoulement dans les égouts et un niveau d'eau sortant du lit naturel du cours d'eau provoque une inondation. La seconde phase, plus qualitative, vise à observer les impacts du barrage en termes de sédimentation et d'obstruction des émissaires d'égouts pluviaux.

\section{Observations}

Une sortie en embarcation sur la rivière a été effectuée le 20 septembre 2011 avec comme premier objectif d'observer les dépôts de sédiments, l'obstruction des conduites pluviales et l'envasement des enrochements. Le second objectif de cette sortie était d'observer des décrochements de sédiments causés par l'ouverture de la vanne de fond, qui a été exceptionnellement ouverte à ce moment pour abaisser la rivière dans le cadre de travaux de réfection de ponts enjambant la rivière.

\section{Simulations}

Les deux logiciels de modélisation HEC-RAS et River2D ont été utilisés conjointement. HECRAS est un logiciel développé pour analyser les écoulements en rivière. Les simulations en régime permanent permettent de visualiser le profil de la surface de l'eau. HEC-RAS prend en compte la géométrie du canal, ainsi que la présence de structures hydrauliques telles que des digues, des barrages, ou encore des ponts. River2D permet de simuler des écoulements torrentiel et fluvial, ainsi que le transport de sédiments, tout comme HEC-RAS d'ailleurs. Les résultats dans River2D permettent de visualiser les vecteurs de vitesse à chaque endroit de la rivière (d'où 
le nom 2D), option qui n'est pas disponible dans HEC-RAS. Dans cette étude, HEC-RAS a été utilisé pour déterminer les hauteurs d'eau sur le tronçon aval et pour estimer les conditions initiales des simulations en régime permanent dans River2D.

L'étude se subdivise en deux thèmes: 1) le comportement hydraulique de la rivière; et 2) le transport de sédiments. L'étude des vitesses et des niveaux d'eau a été réalisée par des simulations de différents scénarios basés sur des débits, des hauteurs des marées, de conditions d'ouverture de la vanne, de frottement et de la présence ou non du barrage. Pour déterminer s'il y a transport de sédiments, il a fallu évaluer l'érosion causée par les vitesses lors des crues en rivière et des marées. Ces variables sont influencées principalement par les débits entrants et sortants du système, par les vitesses obtenues des différentes simulations du comportement hydraulique de la rivière et par les forces tractrices qui y sont associées et par les propriétés des sédiments.

Pour chaque scénario :

1. Une simulation en régime permanent a été réalisée avec HEC-RAS pour évaluer les niveaux d'eau en régime permanent;

2. Une simulation en régime permanent de River2D a été réalisée pour trouver les conditions initiales à l'équilibre dans le modèle River2D ;

3. Une simulation en régime non-permanent a été réalisée dans HEC-RAS pour établir la variation du niveau d'eau en amont du barrage dans le temps selon les marées et;

4. Finalement, en employant les courbes de niveau juste en amont du barrage en fonction du temps générées par HEC-RAS, les simulations en régime non-permanent de River2D ont été réalisées. 
Les variables indépendantes de ce projet sont la hauteur des marées dans le temps, le débit de la rivière Saint-Charles en amont du tronçon à l'étude, les conditions d'ouverture de la vanne, les coefficients de pertes d'énergie par frottement sur les berges et le fond et la présence ou non du barrage. C'est selon différentes conditions imposées à ces variables que les scénarios ont été créés et simulations réalisées.

\section{Marées}

Trois cycles de marée ont été employés dans le cadre des modélisations. Les marées locales du fleuve St-Laurent au niveau de la Ville de Québec sont semi-diurnes et montent typiquement à leur maximum en 5 heures et prennent 7 heures à redescendre. La première marée employée dans les modèles est la marée moyenne de pleine mer, qui atteint 2,934 m en pleine mer supérieure et 1,466 m en pleine mer inférieure dans le référentiel géodésique, présentée à la Figure 2 (Pêches et Océans Canada 2011). La seconde marée à l'étude est la grande marée de pleine mer. Cette marée peut atteindre 4,134 m en pleine mer supérieure et -1,766 m en pleine mer inférieure. Finalement, la troisième marée à l'étude est la marée extrême la plus haute enregistrée à Québec. Elle est le résultat d'une combinaison entre la grande marée, un débit important dans le SaintLaurent et des vents Nord-Ouest dominants qui poussent l'eau vers Québec. Cette marée a atteint 5,134 m. Dans le cadre de cette étude, son minimum a été évalué à $-0,766 \mathrm{~m}$. Les marées de faibles intensités n'ont pas été retenues puisqu'elles avaient peu d'intérêt au niveau des risques d'inondation.

\section{Débits}

Le débit s'écoulant dans la rivière Saint-Charles est la deuxième variable indépendante d'importance pour ce projet après les marées. L'évaluation des débits pour le projet a été difficile 
puisque les mesures colligées par le MDDELCC sur ce tronçon de la rivière Saint-Charles sont incomplètes. Le niveau de l'eau de la rivière Saint-Charles au tronçon à l'étude est en effet modulé par une série de barrages tant sur la rivière Saint-Charles elle-même que sur certains de ses affluents: la rivière Duberger et la rivière Lorette. La seule station hydrométrique sur la rivière Saint-Charles elle-même est située en amont de ces rivières. D'autres stations ont aussi collecté des données sur les rivières Duberger et Lorette, mais le nombre d'années est limité. Finalement, l'occupation du territoire, la forme du bassin versant et les pentes rendent le bassin de la rivière Saint-Charles très hétérogène: forestier en amont et urbanisé en aval, ce qui complique davantage l'emploi de méthodes traditionnelles d'estimation des débits. Pour ce projet, les historiques des débits des rivières Lorette et Duberger, obtenus du CEHQ (2011), ont été comparés avec ceux de la station de mesure de la rivière Saint-Charles pour déterminer quels poids ils avaient dans le débit passant dans le tronçon à l'étude. En période de crue, les données historiques montrent que la rivière Duberger a un débit variant entre $19 \%$ et $23 \%$ du débit à la station hydrométrique de la rivière Saint-Charles. La rivière Lorette, quant à elle, a un débit variant entre $19 \%$ et $31 \%$. En période estivale, ces valeurs diminuent dans le cas des deux rivières jusqu'à moins de $10 \%$. Les mesures de terrain, prises en conditions estivales d'étiage, confirment ces mesures avoisinant les $10 \%$. Dans le cadre de ce projet, des valeurs de $20 \%$ pour la rivière Duberger et de $25 \%$ pour la rivière Lorette ont été retenues pour les périodes de crue et des valeurs de $10 \%$ ont été retenues pour les périodes d'étiage et estivale. Le Tableau 1 résume ces apports : les débits varient de $0,06 \mathrm{~m}^{3} / \mathrm{s}$ dans la Duberger et la Lorette en étiage à $130 \mathrm{~m}^{3} / \mathrm{s}$ en crue maximale pour l'aval de la rivière Saint-Charles.

Quatre débits enregistrés à la station hydrométrique de la rivière Saint-Charles (CEHQ-050904) ont servi à l'étude. Ces débits sont l'étiage, le débit estival médian, le débit de la crue annuelle 
médiane et le débit de la crue maximale enregistrée. Avec les apports respectifs de chaque rivière, le Tableau 1 donne les débits utilisés dans chaque simulation. Les débits sont considérés constants tout au long des simulations puisqu'ils sont basés sur des moyennes quotidiennes enregistrées par le CEHQ.

\section{Conditions d'ouverture de la vanne}

La vanne de fond du barrage, selon sa position ouverte ou fermée, modifie les débits au barrage et les niveaux d'eau. Pour le projet, trois scénarios ont été retenus : 1) la position ouverte, 2) l'ouverture-fermeture et 3) la position fermée.

Dans le premier scénario, la porte reste ouverte en permanence, peu importe la hauteur de la marée. Cette position a pour avantage de permettre aux eaux du fleuve de pénétrer plus profondément dans la rivière et favorise un meilleur échange entre les deux plans d'eau. Dans le deuxième scénario, la vanne s'ouvre et se ferme selon la hauteur d'eau en aval en fonction des marées. Dans ce cas précis, la vanne s'ouvre lorsque le niveau du fleuve passe sous 0,534 m, c'est-à-dire lorsque le niveau passe sous le point le plus élevé de la vanne. Lorsque le niveau d'eau remonte par-dessus la vanne, soit à $0,634 \mathrm{~m}$, celle-ci se ferme. Ce choix de scénario repose sur des aspects de sécurité, pour empêcher les hautes marées de pénétrer dans la rivière. De plus, il favorise une accumulation d'eau en amont du barrage, qui favorisera des vitesses plus fortes lorsque la vanne sera ouverte à nouveau, à cause du niveau d'eau plus élevé en amont. Dans le troisième scénario, la rivière ne peut se déverser dans le Saint-Laurent que par les clapets antimarées situés sur le dessus du barrage. Ce choix de scénario est pour évaluer le comportement de la rivière dans la situation actuelle où la vanne est pratiquement toujours fermée.

Pour les fins de la simulation, la vanne prend 5 minutes à s'ouvrir (un pas de temps). Par la suite, la vanne reste ouverte pour la durée complète de la simulation. La vanne utilise un coefficient de 
contraction de 0,6 lorsque l'écoulement est libre à l'aval et un coefficient de 0,8 lorsque l'écoulement est submergé.

\section{Caractéristiques des simulations}

Les simulations doivent prendre en compte les pertes d'énergie par frottement. Tout écoulement en rivière comporte des pertes d'énergie dues au frottement sur le fond et les berges. Les logiciels de modélisation HEC-RAS et River2D emploient deux valeurs différentes pour évaluer ces pertes par frottement. HEC-RAS emploie le coefficient de Manning ( $n$ ) alors que River2D emploie la hauteur de rugosité équivalente $(h)$. Pour un cours d'eau naturel avec des mouilles, des plantes aquatiques et des enrochements, des valeurs de $n$ se situant entre 0,035 et 0,060, en moyenne 0.045, sont suggérées par Chow (1959). Cependant, pour des cours d'eau ayant une largeur plus élevée que $30 \mathrm{~m}$ en crue, comme c'est le cas de la rivière Saint-Charles, il est préférable de prendre la valeur minimale (Chow, 1959). La valeur retenue dans le cadre de ce projet est de 0,035. Pour une profondeur moyenne supérieure à $1 \mathrm{~m}$, qui est généralement présente sur tout le tronçon à l'étude, il est approprié d'utiliser, pour une valeur de $n$ de 0,035 , une valeur de $h$ de 0,1 m (Dingman 1984). Une étude de sensibilité menée sur les scénarios 1, 2, 3 et 4 montre des différences de moins de 5\% sur les niveaux d'eau selon que la valeur de Manning utilisée est de 0,035 ou 0,045 .

Ensuite, les propriétés hydrauliques des sédiments et des ouvrages en pierres doivent être définis. Les principaux sédiments (ou alluvions) qui s'accumulent dans la rivière peuvent être qualifiés de limons et de sables. Les diamètres effectifs de ces deux catégories de sédiments varient entre 0,002 et $2 \mathrm{~mm}$. Ce sont les sédiments qui s'accumulent et qui doivent être évacués. La matrice de 
pierres utilisées pour les berges dans le projet de renaturalisation des berges a une granulométrie allant de 4 à $250 \mathrm{~mm}$. Il est important d'éviter les vitesses excessives qui pourraient endommager ces ouvrages.

Pour la durée de simulation et le pas de temps, la durée complète des simulations est de 24 heures, soit environ 2 cycles de marées. Le pas de temps normal de la simulation HEC-RAS est de 5 minutes, sauf pour les marées extrêmes qui ont des pas à chaque minute pour un meilleur lissage de la courbe de hauteur d'eau en fonction du temps. Cette courbe, qui est utilisée dans River2D, ne doit pas avoir de trop grandes variations entre chaque pas de temps pour faciliter les calculs. Des résultats exhaustifs sont saisis à toutes les 10 minutes dans HEC-RAS. Les pas de temps de River2D sont variables selon l'équilibre du système puisque le logiciel choisit ses pas de temps pour limiter la variation dans le système entre chaque pas de temps de calcul mais les résultats sont compilés à toutes les 10 minutes.

Enfin, la représentation graphique dans les deux modèles est importante. Le modèle River2D effectue ses calculs selon un maillage. Il est important de bien choisir le maillage car ce choix a un impact sur la capacité de calcul et de résolution du modèle. En effet, un maillage trop serré augmente considérablement le nombre de nœuds à calculer et peut entraîner de longs temps de calcul peu pratiques ou peut provoquer des instabilités dans le logiciel. Le maillage employé est donné à la Figure 3a. Le maillage a été divisé en deux catégories : celles susceptibles d'être dans l'écoulement et celles dans les zones peu susceptibles d'être inondées. Les mailles sont triangulaires et leurs côtés dans la zone d'écoulement (foncée) sont de $5 \mathrm{~m}$ alors qu'ils sont de 30 m à l'extérieur du cours d'eau. Il y a en tout 20147 sommets de maille. La bathymétrie du tronçon dans River2D est présentée à la Figure 3b, qui localise aussi des épis de pierre de 100 à 
$600 \mathrm{~mm}$ de diamètre servant à protéger les berges. Pour le modèle HEC-RAS, les sections définies pour la bathymétrie sont présentées à la Figure $4 \mathrm{a}$ et la Figure $4 \mathrm{~b}$ donne la position et la dimension des clapets anti-marées et de la vanne de fond du barrage Samson.

\section{RÉSULTATS}

\section{Observations sur le terrain concernant la sédimentation}

L'importante sédimentation dans le tronçon à l'étude a pu être observée de manière qualitative lors d'une sortie dans une embarcation sur la rivière le 20 septembre 2011, alors que la vanne de fond était ouverte. Près de Marie-de-l'Incarnation (voir Figure 1), des dépôts d'alluvions ont été recensés sur les deux berges. D’importants décrochements de sédiments, dus aux vitesses augmentées suite à l'ouverture de la vanne, ont été observés dans ce secteur. Légèrement en amont de l'autoroute Laurentienne, un envasement des enrochements et des frayères placés lors du réaménagement des berges réalisé entre 2002 et 2006 a été observé. Tout juste à côté de l'autoroute Laurentienne, les premiers signes d'une obstruction d'émissaire pluvial ont été recensés.

De forts dépôts d'alluvions ont aussi été relevés à l'intersection du ruisseau Lairet, qui se jette dans la rivière Saint-Charles au parc Cartier-Brébeuf, et de la rivière. En aval du parc CartierBrébeuf, la rivière entre dans sa partie la plus affectée par le barrage. Les dépôts sédimentaires sont beaucoup plus importants et le décrochement des berges dû à l'ouverture de la vanne de fond est plus facilement observable. Il est à noter que ce tronçon a été renaturalisé en 2007; les sédiments observés s'y sont accumulés sur plus d'un mètre d'épaisseur en moins de 4 ans (Figure 5a). Près de l'autoroute Dufferin-Montmorency, à moins de 200 mètres en amont du barrage, il a été possible d'observer un énorme banc de dépôts organiques qui longe la berge Nord. Ces dépôts 
sont peu compactés et riches en gaz de décomposition (Figure 5b). En résumé, à mesure que l'embarcation s'approchait du barrage Samson, le volume des sédiments déposés augmentait tout comme les signes de décrochement.

Des mesures ont été réalisées en conditions de faible débit en septembre 2011 avec les vannes ouvertes. Des mesures de débits à l'ADV (Flowtracker de SonTek) sur la rivière Duberger, la rivière Lorette et dans la partie amont du modèle sur la rivière Saint-Charles ont permis de vérifier la distribution des débits et de calibrer le modèle pour les faibles débits. Des observations sur le terrain ont aussi été réalisées en condition de crue s'apparentant au débit de la crue médiane en mars 2012. À ce moment, la rivière était en décrue d'une crue majeure et le débit moyen journalier à la station de la rivière Saint-Charles était d'environ $45 \mathrm{~m}^{3} / \mathrm{s}$ le jour de la prise des observations. Les niveaux relevés (à quelques centimètres de la piste cyclable sous le pont Mariede-l'Incarnation, submersion des îles et à moins de $600 \mathrm{~mm}$ de la piste cyclable dans ce secteur, au plein bord dans la portion des épis et à moins de $400 \mathrm{~mm}$ d'un débordement juste en amont de la rue du Pont) correspondaient aux niveaux modélisés pour des crues similaires.

\section{Simulations}

Le Tableau 2 présente un descriptif des 24 scénarios simulés ainsi qu'une appréciation du risque d'inondation de la rivière hors berge (sécurité des citoyens et des biens), de la vitesse moyenne estimée (entraînement des sédiments) sur le tronçon en amont et en aval du parc Cartier-Brébeuf et des échanges d'eau (apport d'oxygène dissous) avec le fleuve Saint-Laurent. Les risques d'inondation sont soit «Faibles», importants sur le «Secteur des îles», importants sur le «Secteur des îles et parc Cartier-Brébeuf» ou «Généralisés» sur tout le tronçon à l'étude. Les vitesses 
varient de très faibles (moins de $0,5 \mathrm{~m} / \mathrm{s}$ ) à $2,5 \mathrm{~m} / \mathrm{s}$ et les échanges avec le fleuve varient de «Aucun» ou «Seulement près du barrage» à «Généralisés».

Tel que mentionné précédemment, la gestion actuelle du barrage à vanne fermée et clapets antimarées crée un plan d'eau favorisant la sédimentation et diminuant de façon importante les échanges d'eau avec le fleuve. Les observations sur le terrain confirment que l'accumulation de sédiments est importante en amont du barrage Samson et qu'elle diminue la capacité d'évacuation des réseaux d'égout. Les observations confirment aussi que l'accumulation de sédiments menace les écosystèmes par l'envasement des frayères et des plantes (Figure 5).

La gestion des débits au barrage Samson présente, selon les 24 simulations réalisées, des avantages certains pour minimiser ces problèmes. Tout d'abord, il faut comparer les vitesses obtenues et les échanges d'eau avec le fleuve Saint-Laurent en débit d'étiage $\left(0,8 \mathrm{~m}^{3} / \mathrm{s}\right)$ et vanne ouverte (scénarios 1, 5, 9 et 13) par rapport à la simulation 21 (même débit mais vanne fermée) (Figures $6 \mathrm{a}, 6 \mathrm{~b}$ et $6 \mathrm{c}$ ). Dans les 4 simulations avec ouverture de la vanne, des vitesses propices à un entraînement des sédiments (de taille $0,005 \mathrm{~mm}$ à $5 \mathrm{~mm}$ ) ont été obtenues. L'avantage qui reste cependant le plus important est l'échange des eaux sur tout le tronçon à l'étude avec celles du fleuve Saint-Laurent. En effet, les eaux moins oxygénées de la rivière Saint-Charles sont remplacées par les eaux généralement plus froides et mieux oxygénées du fleuve. Il y a donc des gains majeurs à faire avec l'ouverture de la vanne en période d'étiage. Les seuls problèmes qui peuvent être soulevés par l'ouverture des vannes consistent en des problèmes d'esthétisme et d'usage. Avec les vannes ouvertes, l'aspect de la rivière change, les niveaux d'eau sont plus bas et le chenal se rétrécit, dénudant des zones de sédimentation, ce qui pourrait amener une opposition citoyenne, alors que d'autres personnes pourraient préférer cet aspect plus naturel de 
la rivière. Certains usages récréatifs sont restreints car il devient difficile de pratiquer la navigation de plaisance lorsque les niveaux d'eau sont plus bas. Il faudrait penser à des solutions hybrides comme des ouvertures contrôlées au lieu de permanentes. Il pourrait cependant y avoir un gain au niveau de la pêche grâce à une augmentation du nombre d'espèces présentes.

Les gains deviennent plus importants dans les simulations à débit estival $\left(8 \mathrm{~m}^{3} / \mathrm{s}\right)$. Par rapport à la simulation 22 à vanne fermée, les simulations 2, 6, 10 et 14 offrent toutes des vitesses favorisant un bon entraînement du limon déposé. Les échanges restent aussi très bons avec le Saint-Laurent, atteignant le parc Cartier-Brébeuf dans les scénarios d'ouverture/fermeture de la vanne à marée haute (scénarios 13 et 14) et Marie-de-l'Incarnation dans les autres cas. Cependant, les usages et l'esthétique du cours d'eau sont affectés de la même façon que dans les simulations au débit d'étiage.

Il a été démontré que l'ouverture de la vanne lors des crues médianes de $58 \mathrm{~m}^{3} / \mathrm{s}$ modélisées aux scénarios 3, 7, 11, 15, 17 et 19 pouvait aider à évacuer plus d'eau qu'une gestion en ouverturefermeture ou en scénario avec vanne fermée uniquement. En effet, le niveau d'eau plus élevé en amont qu'en aval du barrage fait que les marées ne peuvent entrer dans le plan d'eau et c'est plutôt l'eau en amont du barrage qui s'évacue. La fermeture de la vanne contribue à augmenter le niveau des eaux et peut même être problématique, tel que démontré dans les cas 15 et 17 où le haut niveau d'eau causé par le barrage posait des risques d'inondation dans le secteur des îles.

Lors des crues extrêmes, des inondations dues à des niveaux supérieurs à $5 \mathrm{~m}$ sont à prévoir dans tous les scénarios, mais ceux-ci sont accentués lors des scénarios à vanne fermée ou en ouverturefermeture. Cela est dû principalement au fait que, lorsque la vanne est fermée et la marée est 
haute, l'eau ne peut presque plus s'évacuer. Le débit considérable de $130 \mathrm{~m}^{3} / \mathrm{s}$ provoque une élévation du niveau d'eau en amont du barrage, causant les inondations. Dans ces cas extrêmes, les clapets anti-marées peuvent même contribuer au problème. La simulation 20 - sans barrage, par exemple, présente moins de problèmes que les simulations 16 et 18 avec vanne en ouverturefermeture et des marées fortes ou extrêmes. Il faut, en effet, noter que les cas 16 et 18 n'ont pu être menés normalement car le niveau d'eau devient trop important et cause des inondations majeures, ce qui cause des problèmes de convergence aux deux modèles. Les simulations ont donc été menées sans la présence des clapets anti-marées.

Le barrage a son utilité pour prévenir les inondations lors des fortes marées et marées extrêmes, mais cet effet peut être néfaste si des crues exceptionnelles accompagnent ces marées. L'ouverture de la vanne de fond est bénéfique dans la majorité des scénarios. Seuls des scénarios d'ouverture de la vanne avec marée extrême et débits estivaux ou d'étiage ont quelques aspects négatifs au niveau des usages et de l'esthétisme. L'ouverture-fermeture serait par contre une bonne solution pour ces cas. La véritable limitation pour l'ouverture de la vanne réside plutôt dans l'aspect esthétique et au niveau de l'acceptabilité sociale. Finalement, les observations sur le terrain indiquent un danger réel lié au rehaussement des niveaux d'eau, qui cause l'accumulation annuelle de sédiments. L'ouverture de la vanne réduit l'ampleur de ce phénomène.

\section{CONCLUSION}

Les simulations ont permis de démontrer que, dans des cas extrêmes, le barrage peut constituer un risque pour les inondations. Il a aussi été observé que le barrage créait bel et bien des conditions idéales pour la sédimentation presqu'à tous les moments sauf en périodes de crues extrêmes. La sédimentation créée par le barrage a aussi été observée sur le terrain et elle constitue 
une menace pour les écosystèmes et un problème au niveau des émissaires d'égout. Le barrage a, par contre, pour effet de protéger l'amont lors de fortes marées à débits plus faibles.

L'évaluation par modélisation a démontré que l'ouverture de la vanne de fond, dans la majeure partie des cas, avait des effets bénéfiques en permettant un entraînement des sédiments et une évacuation des eaux dans les conditions les plus extrêmes. L'ouverture à marée basse et la fermeture à marée haute n'ont pas donné de résultats bien différents de l'ouverture permanente, sinon qu'elle est plus risquée pour les inondations dans les périodes de crues et plus sécuritaire lors de la période estivale. L'entraînement des sédiments et les échanges d'eau avec le SaintLaurent auront pour avantages de donner de meilleures conditions pour les espèces aquatiques tant au niveau de l'oxygène dissous qu'au niveau du bon dégagement des frayères.

La gestion des débits par la vanne de fond permettrait, selon les simulations, de diminuer les risques d'inondation et pourraient améliorer les conditions pour les espèces aquatiques présentes dans la rivière. Il faudra par contre développer des solutions pour atténuer les impacts négatifs sur l'esthétique et les usages récréatifs qui seront perdus si la vanne est ouverte en permanence. Étant donné que la vanne doit être changée, il y a une opportunité de déterminer, dans un choix de société, s'il est préférable que la rivière redevienne naturelle avec l'ouverture permanente de la vanne. Comme tout problème relié à la gestion de l'eau, des compromis sont nécessaires entre les aspects économique, social et écologique.

\section{REMERCIEMENTS}

Merci à François Proulx du Service de l'environnement de la Ville de Québec pour son temps et Julie Garneau et Diane Arjoon pour l'édition de l'article. 


\section{RÉFÉRENCES}

Alibert, M., Assani, A.A., Gratton, D, Leroux, D., and Laurence, M. 2011. Statistical analysis of the evolution of a semialluvial stream channel upstream from an inversion-type reservoir: The case of the Matawin River (Quebec, Canada). Geomorphology 131 :28-34.

Assani A.A., Buffin-Bélanger, T., et Roy, A.G. 2002. Analyse d'impacts d'un barrage sur le régime hydrologique de la rivière Matawin (Québec, Canada). Revue des Sciences de l'Eau 15 (2) : 557-574. DOI : 10.7202/705469ar

Assani, A.A., Gravel, É., Buffin-Bélanger, T., et Roy, A.G. 2005. Impacts des barrages sur les débits annuels minimums en fonction des régimes hydrologiques artificialisés au Québec (Canada). Revue des Sciences de l'Eau 18 (1) : 103-127. DOI: 10.7202/705552ar

CEHQ. 2011. Niveaux et débits. Centre d'expertise hydrique du Québec. Canada. Disponible à : http://www.cehq.gouv.qc.ca/hydrometrie/index.htm [consulté en novembre 2011].

Chow, Ven Te. 1959. Open-Channel Hydraulics. University of Illinois. États-Unis.

Compagnon, N. 2000. Étude de faisabilité d'un échange d'eau entre le fleuve Saint-Laurent et la rivière Saint-Charles. École des Ingénieurs de Paris, Paris, France.

Dingman, S.L. 1984. Fluvial Hydrology. W H Freeman \& Co (Sd). University of New Hampshire. États-Unis.

Lick, W. 2009. Sediment and Contaminant Transport in Surface Waters. CRC Press, États-Unis. MDDELCC. 2002. Critère de qualité de l'eau de surface. Disponible à : http://www.mddelcc.gouv.qc.ca/eau/criteres eau/details.asp?code=S0365 [consulté en août 2014].

Pêches et Océans Canada. 2011. Informations sur les marées à Québec (Lauzon). Service $\begin{array}{llll}\text { Hydrographique } & \text { du } & \text { Canada. } & \text { Disponible }\end{array}$ http://www.niveauxdeau.gc.ca/fra/station?sid=3250 [consulté en novembre 2011]. 
Sentinelles de la rivière Petitcodiac. 2013. Les 10 pires sources de pollution de l'écosystème de la Petitcodiac en 2012. Disponible en ligne à : http://petitcodiac.org/wpcontent/uploads/2013/11/10 pires sources_2012_FR_v2.pdf [consulté en août 2014].

Sentinelles de la rivière Petitcodiac. 2014. Impact du pont-chaussée de la Petitcodiac. Disponible en ligne à : http://petitcodiac.org/campaigns-2/la-chaussee-petitcodiac/impacts-du-pontchaussee-de-la-petitcodiac/?lang=fr [consulté en juillet 2014].

Société de la rivière Saint-Charles. 2007. La rivière Saint-Charles: un écosystème en renaissance - Étude de faisabilité : Optimisation de la rivière Saint-Charles pour l'ichtyofaune du SaintLaurent. Rapport présenté au Programme Interactions Communautaires. 29 pages.

Steffler, P., and Blackburn, J. 2002. River2D: Two-Dimensional Depth Averaged Model of River Hydrodynamics and Fish Habitat. University of Alberta, Canada.

Syvitski, P.M., Vörösmarty, C.J., Kettner, A.J., and Pamela, G. 2005. Impact of Humans on the Flux of Terrestrial Sediment to the Global Coastal Ocean. Science, New Series 308 (5720) : 376-380. DOI: 10.1126/science.1109454

U.S. Army Corps of Engineers. 2010. HEC-RAS River analysis system User Manual. Hydrologic Engineering Center. Californie.

Ville de Québec. 2012. Suivi de 1'état de la partie aval de rivière Saint-Charles lors de 1'ouverture de la vanne de fond du barrage Samson. Rapport de stage d'Imane Soltani. 28 pages.

Yin, X.A. 2010. Optimizing environmental flows below dams. River Research and Applications, 28(6), 703-716. DOI: 10.1002/rra.1477. 


\section{TABLEAUX}

Tableau 1 - Débits à l'étude en $\mathrm{m}^{3} / \mathrm{s}$

\begin{tabular}{|l|c|c|c|c|c|}
\hline & $\begin{array}{c}\text { Saint-Charles } \\
\text { jaugé }\end{array}$ & Duberger & Lorette & $\begin{array}{c}\text { Total estimé de la } \\
\text { Saint-Charles }\end{array}$ & $\begin{array}{c}\text { Débit choisi pour } \\
\text { simulation }\end{array}$ \\
\hline Étiage & 0,6 & 0,06 & 0,06 & 0,72 & 0,8 \\
\hline Estival moyen & 6,6 & 0,66 & 0,66 & 7,92 & 8 \\
\hline Crue médiane & 40 & 8 & 10 & 58 & 58 \\
\hline Crue maximale & 90 & 18 & 22 & 130 & 130 \\
\hline
\end{tabular}


Tableau 2 - Tableau synthèse des résultats. Les cases en gris représentent les situations les moins souhaitables dues à un fort risque d'inondations, une trop faible vitesse de l'eau ou échange minimal avec le fleuve.

\begin{tabular}{|c|c|c|c|c|c|c|c|}
\hline \# & Débit & Marée & Vanne & $\begin{array}{l}\text { Risque } \\
\text { d'inonda- } \\
\text { tions }\end{array}$ & $\begin{array}{l}\text { Vitesse en } \\
\text { amont du } \\
\text { parc Cartier- } \\
\text { Brébeuf }\end{array}$ & $\begin{array}{c}\text { Vitesse en } \\
\text { aval du } \\
\text { parc } \\
\text { Cartier- } \\
\text { Brébeuf }\end{array}$ & $\begin{array}{c}\text { Échanges } \\
\text { avec le fleuve }\end{array}$ \\
\hline 1 & $\begin{array}{l}\text { Étiage } \\
0,8 \mathrm{~m}^{3} / \mathrm{s}\end{array}$ & \multirow{8}{*}{ Moyenne } & \multirow{4}{*}{ Ouverte } & Faible & $\begin{array}{c}\text { Moins de } \\
0,5 \mathrm{~m} / \mathrm{s}\end{array}$ & $\begin{array}{l}\text { De } 0,5 \mathrm{~m} / \mathrm{s} \\
\text { et } 1,5 \mathrm{~m} / \mathrm{s}\end{array}$ & Généralisé \\
\hline 2 & $\begin{array}{c}\text { Été moyen } \\
8 \mathrm{~m}^{3} / \mathrm{s}\end{array}$ & & & Faible & $\begin{array}{c}\text { Moins de } 1 \\
\mathrm{~m} / \mathrm{s}\end{array}$ & $\begin{array}{c}\text { De } 1 \mathrm{~m} / \mathrm{s} \text { à } \\
1,8 \mathrm{~m} / \mathrm{s}\end{array}$ & Généralisé \\
\hline 3 & $\begin{array}{c}\text { Crue médiane } \\
58 \mathrm{~m}^{3} / \mathrm{s}\end{array}$ & & & Faible & $\begin{array}{c}\text { De } 1 \mathrm{~m} / \mathrm{s} \text { à } \\
1,5 \mathrm{~m} / \mathrm{s}\end{array}$ & $\begin{array}{c}\text { De } 1 \mathrm{~m} / \mathrm{s} \text { à } \\
1,5 \mathrm{~m} / \mathrm{s}\end{array}$ & $\begin{array}{l}\text { Seulement } \\
\text { près du } \\
\text { barrage } \\
\end{array}$ \\
\hline 4 & $\begin{array}{c}\text { Crue extrême } \\
130 \mathrm{~m}^{3} / \mathrm{s}\end{array}$ & & & $\begin{array}{l}\text { Secteur des } \\
\text { Îles }\end{array}$ & $\begin{array}{c}\text { De } 1,2 \mathrm{~m} / \mathrm{s} \text { à } \\
2 \mathrm{~m} / \mathrm{s}\end{array}$ & $\begin{array}{c}\text { De } 1,2 \mathrm{~m} / \mathrm{s} \\
\text { à } 2,4 \mathrm{~m} / \mathrm{s}\end{array}$ & Aucun \\
\hline 5 & $\begin{array}{c}\text { Étiage } \\
0,8 \mathrm{~m}^{3} / \mathrm{s}\end{array}$ & & \multirow{4}{*}{$\begin{array}{l}\text { Ouvertu- } \\
\text { re/ } \\
\text { Fermetu- } \\
\text { re }\end{array}$} & Faible & $\begin{array}{c}\text { Moins de } \\
0,5 \mathrm{~m} / \mathrm{s}\end{array}$ & $\begin{array}{c}\text { De } 0,5 \mathrm{~m} / \mathrm{s} \\
\text { à } 1,5 \mathrm{~m} / \mathrm{s}\end{array}$ & Généralisé \\
\hline 6 & $\begin{array}{c}\text { Été moyen } \\
8 \mathrm{~m}^{3} / \mathrm{s}\end{array}$ & & & Faible & $\begin{array}{c}\text { Moins de } 1 \\
\mathrm{~m} / \mathrm{s}\end{array}$ & $\begin{array}{c}\text { De } 1 \mathrm{~m} / \mathrm{s} \text { à } \\
1,5 \mathrm{~m} / \mathrm{s}\end{array}$ & Généralisé \\
\hline 7 & $\begin{array}{c}\text { Crue médiane } \\
58 \mathrm{~m}^{3} / \mathrm{s}\end{array}$ & & & Faible & $\begin{array}{c}\text { De } 0,8 \mathrm{~m} / \mathrm{s} \text { à } \\
1,2 \mathrm{~m} / \mathrm{s}\end{array}$ & $\begin{array}{c}\text { De } 0,8 \mathrm{~m} / \mathrm{s} \\
\text { à } 1,6 \mathrm{~m} / \mathrm{s}\end{array}$ & Aucun \\
\hline 8 & $\begin{array}{c}\text { Crue extrême } \\
130 \mathrm{~m}^{3} / \mathrm{s}\end{array}$ & & & $\begin{array}{l}\text { Secteur des } \\
\text { Îles }\end{array}$ & $\begin{array}{c}\text { De } 1,2 \mathrm{~m} / \mathrm{s} \text { à } \\
1,8 \mathrm{~m} / \mathrm{s}\end{array}$ & $\begin{array}{c}\text { De } 1,2 \mathrm{~m} / \mathrm{s} \\
\text { à } 2,4 \mathrm{~m} / \mathrm{s}\end{array}$ & Aucun \\
\hline 9 & $\begin{array}{c}\text { Étiage } \\
0,8 \mathrm{~m}^{3} / \mathrm{s}\end{array}$ & \multirow{7}{*}{ Forte } & \multirow{4}{*}{ Ouverte } & Faible & $\begin{array}{c}\text { Moins de } \\
0,5 \mathrm{~m} / \mathrm{s}\end{array}$ & $\begin{array}{c}\text { De } 0,8 \mathrm{~m} / \mathrm{s} \\
\text { à } 1,8 \mathrm{~m} / \mathrm{s}\end{array}$ & Généralisé \\
\hline 10 & $\begin{array}{c}\text { Été moyen } \\
8 \mathrm{~m}^{3} / \mathrm{s}\end{array}$ & & & Faible & $\begin{array}{l}\text { Moins de } \\
1,2 \mathrm{~m} / \mathrm{s}\end{array}$ & $\begin{array}{c}\text { De } 1 \mathrm{~m} / \mathrm{s} \text { à } \\
2 \mathrm{~m} / \mathrm{s}\end{array}$ & Généralisé \\
\hline 11 & $\begin{array}{l}\text { Crue médiane } \\
58 \mathrm{~m}^{3} / \mathrm{s}\end{array}$ & & & $\begin{array}{l}\text { Secteur des } \\
\text { Îles - léger }\end{array}$ & $\begin{array}{c}\text { De } 0,8 \mathrm{~m} / \mathrm{s} \text { à } \\
1,2 \mathrm{~m} / \mathrm{s}\end{array}$ & $\begin{array}{c}\text { De } 1 \mathrm{~m} / \mathrm{s} \text { à } \\
2 \mathrm{~m} / \mathrm{s}\end{array}$ & $\begin{array}{l}\text { Seulement } \\
\text { près du } \\
\text { barrage }\end{array}$ \\
\hline 12 & $\begin{array}{c}\text { Crue extrême } \\
130 \mathrm{~m}^{3} / \mathrm{s}\end{array}$ & & & $\begin{array}{l}\text { Secteur des } \\
\text { Îles }\end{array}$ & $\begin{array}{c}\text { De } 1 \mathrm{~m} / \mathrm{s} \text { à } \\
1,5 \mathrm{~m} / \mathrm{s}\end{array}$ & $\begin{array}{c}\text { De } 1,5 \mathrm{~m} / \mathrm{s} \\
\text { à } 2,5 \mathrm{~m} / \mathrm{s}\end{array}$ & Aucun \\
\hline 13 & $\begin{array}{l}\text { Étiage } \\
0,8 \mathrm{~m}^{3} / \mathrm{s}\end{array}$ & & \multirow{3}{*}{$\begin{array}{l}\text { Ouvertu- } \\
\text { re/ } \\
\text { Fermetu- } \\
\text { re }\end{array}$} & Faible & $\begin{array}{c}\text { Moins de } \\
0,5 \mathrm{~m} / \mathrm{s}\end{array}$ & $\begin{array}{c}\text { De } 0,8 \mathrm{~m} / \mathrm{s} \\
\text { à } 1,8 \mathrm{~m} / \mathrm{s}\end{array}$ & Généralisé \\
\hline 14 & $\begin{array}{c}\text { Été moyen } \\
8 \mathrm{~m}^{3} / \mathrm{s}\end{array}$ & & & Faible & $\begin{array}{c}\text { Moins de } \\
1,2 \mathrm{~m} / \mathrm{s}\end{array}$ & $\begin{array}{c}\text { De } 1 \mathrm{~m} / \mathrm{s} \text { à } \\
2 \mathrm{~m} / \mathrm{s}\end{array}$ & Généralisé \\
\hline 15 & $\begin{array}{c}\text { Crue médiane } \\
58 \mathrm{~m}^{3} / \mathrm{s}\end{array}$ & & & $\begin{array}{c}\text { Secteur des } \\
\text { Îles et parc } \\
\text { Cartier- } \\
\text { Brébeuf }\end{array}$ & $\begin{array}{c}\text { De } 0,9 \mathrm{~m} / \mathrm{s} \text { à } \\
1,5 \mathrm{~m} / \mathrm{s}\end{array}$ & $\begin{array}{c}\text { De } 1 \mathrm{~m} / \mathrm{s} \text { à } \\
2 \mathrm{~m} / \mathrm{s}\end{array}$ & Aucun \\
\hline
\end{tabular}




\begin{tabular}{|c|c|c|c|c|c|c|c|}
\hline 16 & $\begin{array}{c}\text { Crue extrême } \\
130 \mathrm{~m}^{3} / \mathrm{s}\end{array}$ & & & Généralisé & $\begin{array}{c}\text { De } 1,2 \mathrm{~m} / \mathrm{s} \text { à } \\
1,5 \mathrm{~m} / \mathrm{s}\end{array}$ & $\begin{array}{c}\text { De } 1,2 \mathrm{~m} / \mathrm{s} \\
\text { à } 2 \mathrm{~m} / \mathrm{s}\end{array}$ & Aucun \\
\hline 17 & $\begin{array}{l}\text { Crue médiane } \\
58 \mathrm{~m}^{3} / \mathrm{s}\end{array}$ & \multirow{4}{*}{ Extrême } & \multirow{2}{*}{$\begin{array}{l}\text { Ouvertu- } \\
\text { re/ } \\
\text { Fermetu- } \\
\text { re }\end{array}$} & $\begin{array}{c}\text { Secteur des } \\
\text { Îles et parc } \\
\text { Cartier- } \\
\text { Brébeuf }\end{array}$ & $\begin{array}{c}\text { De } 1,2 \mathrm{~m} / \mathrm{s} \text { à } \\
1,5 \mathrm{~m} / \mathrm{s}\end{array}$ & $\begin{array}{c}\text { De } 1,2 \mathrm{~m} / \mathrm{s} \\
\text { à } 2 \mathrm{~m} / \mathrm{s}\end{array}$ & Aucun \\
\hline 18 & $\begin{array}{c}\text { Crue extrême } \\
130 \mathrm{~m}^{3} / \mathrm{s}\end{array}$ & & & Généralisé & $\begin{array}{c}\text { De } 0,8 \mathrm{~m} / \mathrm{s} \text { à } \\
1,8 \mathrm{~m} / \mathrm{s}\end{array}$ & $\begin{array}{c}\text { De } 1 \mathrm{~m} / \mathrm{s} \text { à } \\
2 \mathrm{~m} / \mathrm{s}\end{array}$ & Aucun \\
\hline 19 & $\begin{array}{c}\text { Crue médiane } \\
58 \mathrm{~m}^{3} / \mathrm{s}\end{array}$ & & \multirow{2}{*}{$\begin{array}{c}\text { Sans } \\
\text { barrage }\end{array}$} & Généralisé & - & - & Généralisé \\
\hline 20 & $\begin{array}{c}\text { Crue extrême } \\
130 \mathrm{~m}^{3} / \mathrm{s}\end{array}$ & & & Généralisé & - & - & Généralisé \\
\hline 21 & $\begin{array}{c}\text { Étiage } \\
0,8 \mathrm{~m}^{3} / \mathrm{s}\end{array}$ & \multirow{4}{*}{ Moyenne } & \multirow{4}{*}{ Fermée } & Faible & $\begin{array}{c}\text { Moins de } \\
0,1 \mathrm{~m} / \mathrm{s}\end{array}$ & $\begin{array}{c}\text { Moins de } \\
0,1 \mathrm{~m} / \mathrm{s}\end{array}$ & Aucun \\
\hline 22 & $\begin{array}{c}\text { Été moyen } \\
8 \mathrm{~m}^{3} / \mathrm{s}\end{array}$ & & & Faible & $\begin{array}{c}\text { Moins de } \\
0,3 \mathrm{~m} / \mathrm{s}\end{array}$ & $\begin{array}{c}\text { Moins de } \\
0,2 \mathrm{~m} / \mathrm{s}\end{array}$ & Aucun \\
\hline 23 & $\begin{array}{c}\text { Crue médiane } \\
58 \mathrm{~m}^{3} / \mathrm{s}\end{array}$ & & & Faible & $\begin{array}{c}\text { Moins de } \\
1,2 \mathrm{~m} / \mathrm{s}\end{array}$ & $\begin{array}{c}\text { Moins de } \\
0,8 \mathrm{~m} / \mathrm{s}\end{array}$ & Aucun \\
\hline 24 & $\begin{array}{l}\text { Crue extrême } \\
130 \mathrm{~m}^{3} / \mathrm{s}\end{array}$ & & & $\begin{array}{l}\text { Secteur des } \\
\text { Îles }\end{array}$ & $\begin{array}{c}\text { De } 0,6 \mathrm{~m} / \mathrm{s} \text { à } \\
1,4 \mathrm{~m} / \mathrm{s}\end{array}$ & $\begin{array}{c}\text { De } 0,6 \mathrm{~m} / \mathrm{s} \\
\text { à } 1,2 \mathrm{~m} / \mathrm{s}\end{array}$ & Aucun \\
\hline
\end{tabular}




\section{LÉGENDE DES FIGURES}

Figure 1 - Secteur à l'étude et zones de la rivière Saint-Charles sous l'influence des marées en l'absence du barrage Samson. Les zones vertes représentent les marais et les zones brunes correspondent aux marécages.

Figure 2 - Marée à la Ville de Québec.

Figure 3 - Modèle River2D. a) Maillage : petites mailles de 5 mètres, grandes de 30 mètres; b) Bathymétrie du tronçon.

Figure 4 - Modèle HEC-RAS. a) Sections définies; b) Dimensions du barrage, des clapets antimarées et de la vanne de fond.

Figure 5 - a) Observations d'épaisse accumulation et décrochement en aval du parc CartierBrébeuf; b) Dépôts en amont de l'autoroute Dufferin-Montmorency.

Figure 6 - Résultats dans River-2D des vitesses maximales. a) Scénario 1 : débit d'étiage, à marée moyenne et vanne ouverte. b) Scénario 9: débit d'étiage, à marée forte et vanne ouverte. c) Scénario 21 : régime permanent, débit d'étiage, vanne fermée. 


\begin{abstract}
The Joseph-Samson Dam is located on the St. Charles River near the confluence with the St. Lawrence River in the Lower Town of Quebec City. The dam creates a $3.7 \mathrm{~km}$ long reservoir and, with its anti-tidal gates, protects from flooding during very high spring tides in the St. Lawrence. However, the dam has completely changed the formerly natural estuary into an unsightly decantation basin formed from the sediment influx coming from the watershed. Highly productive fish runs up the St. Charles are a thing of the past and significant reduction in dissolved oxygen is now often observed. Furthermore, the dam may actually increase the risk of flooding in the Lower Town under high flows. Onedimensional simulations (HEC-RAS) were performed to assess the dam's impact on flood risks for different bottom gate settings and two-dimensional simulations (RIVER 2D) were performed to assess the dam's impact on river velocities.
\end{abstract}




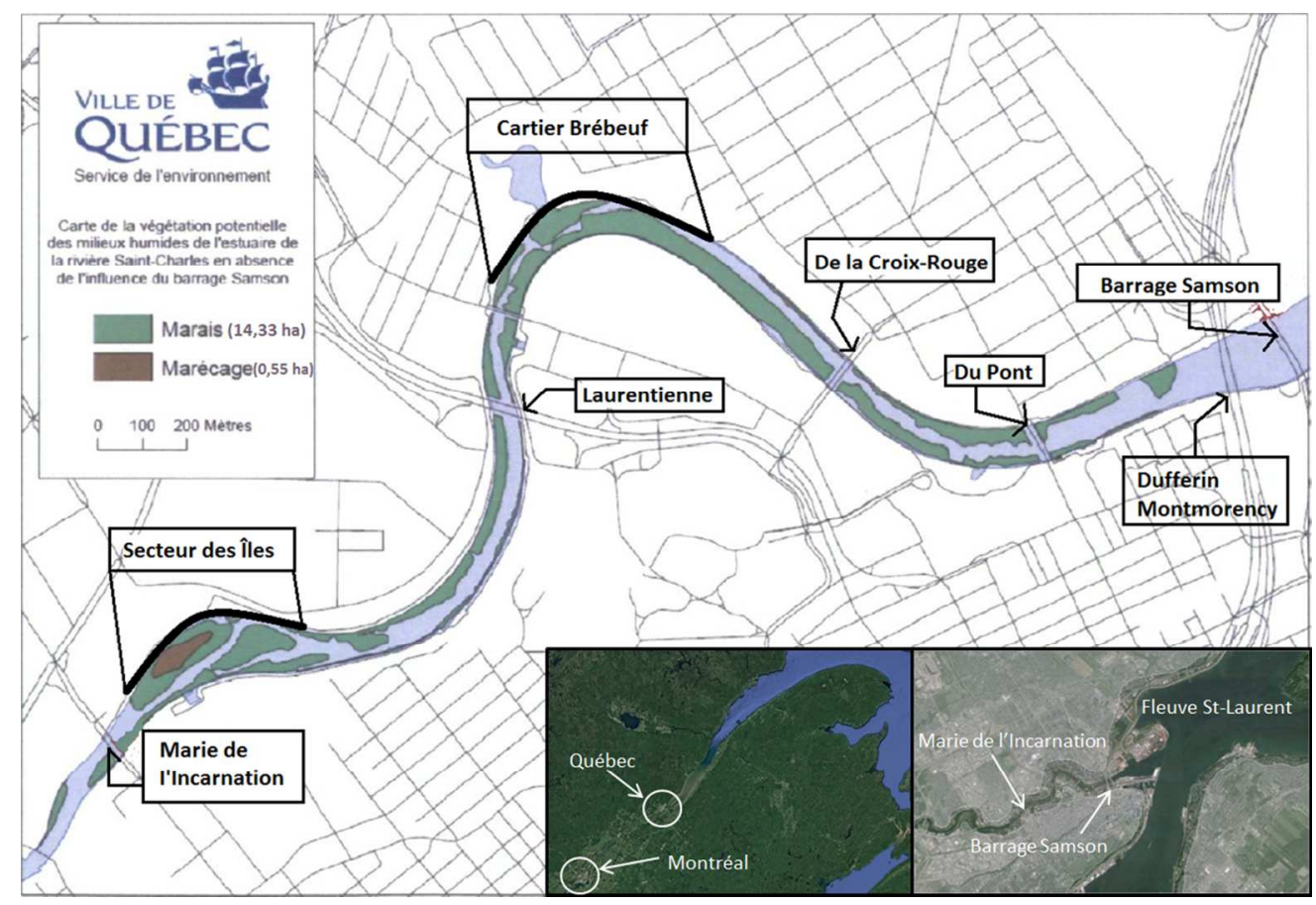

$327 \times 224 \mathrm{~mm}(96 \times 96$ DPI $)$ 


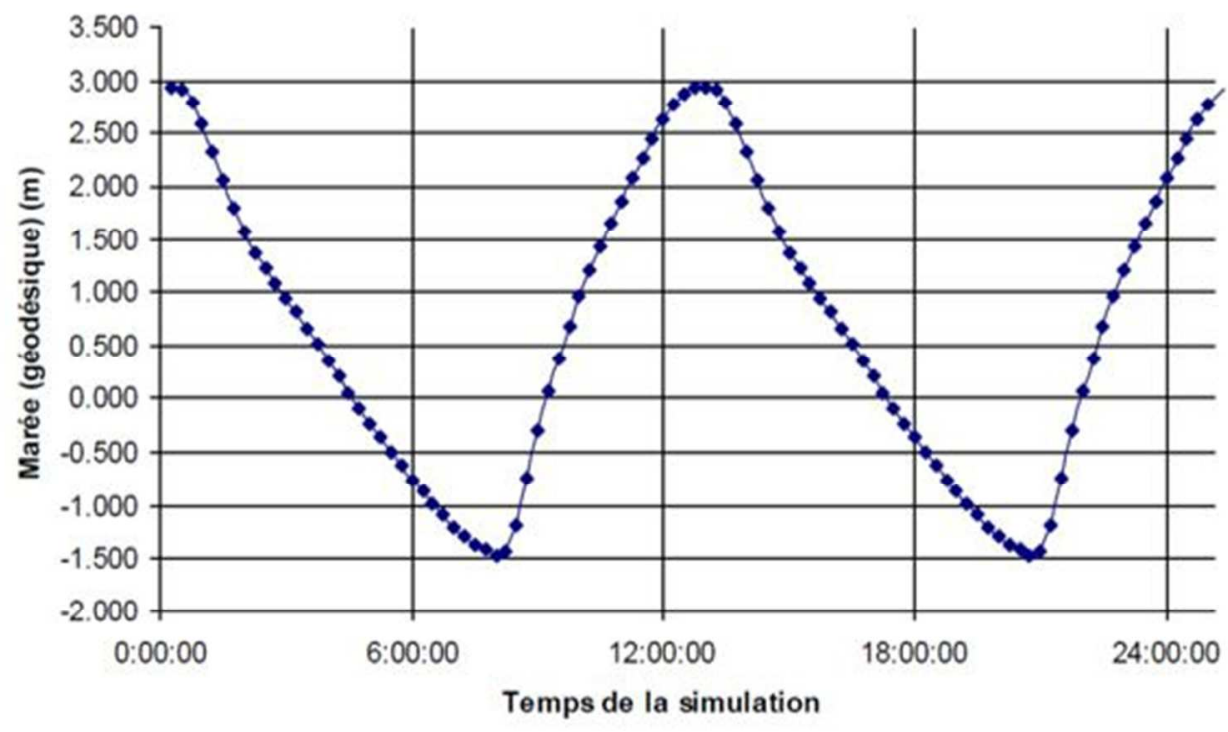

$158 \times 91 \mathrm{~mm}(96 \times 96 \mathrm{DPI})$ 


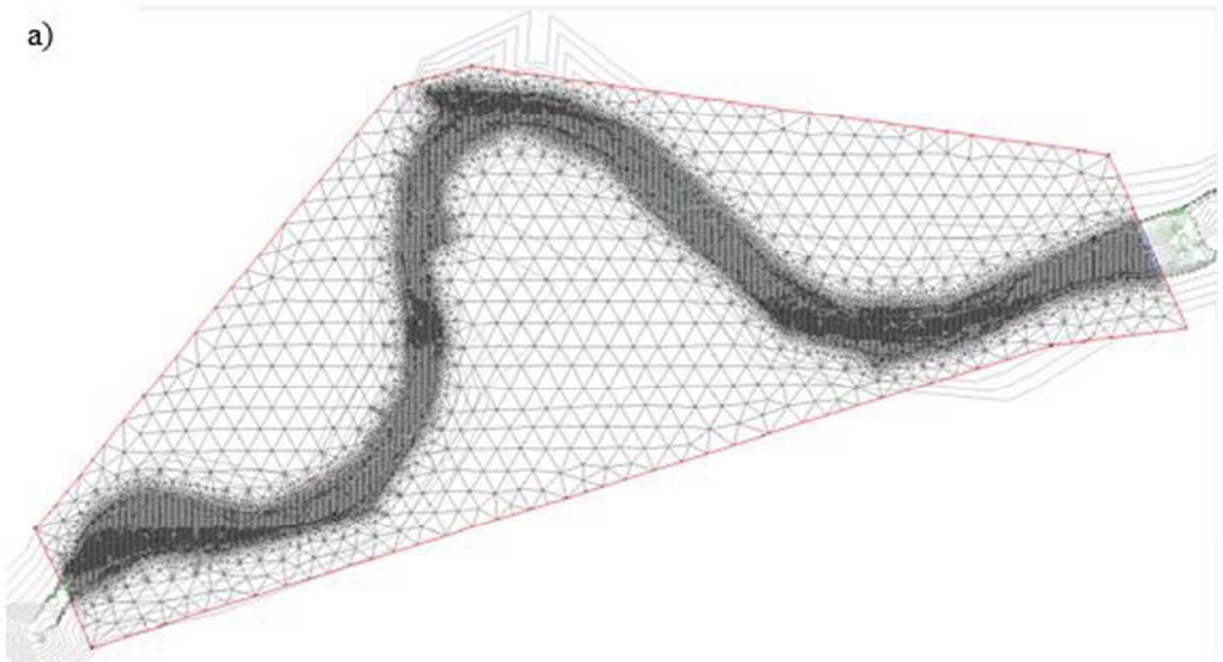

b)

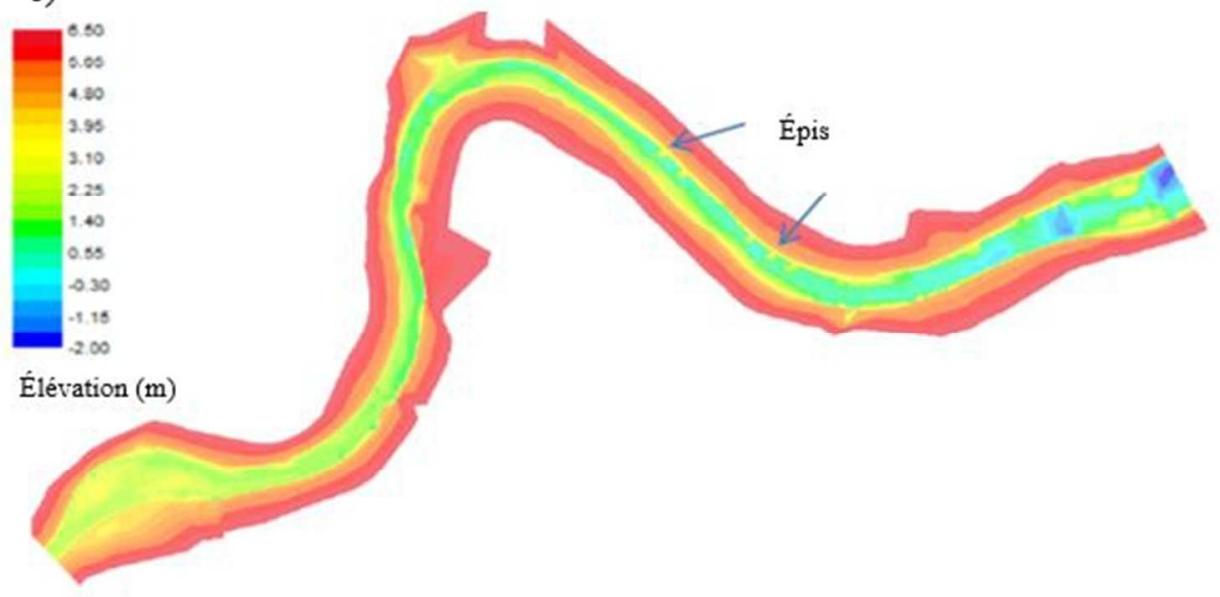

$161 \times 169 \mathrm{~mm}(96 \times 96 \mathrm{DPI})$ 

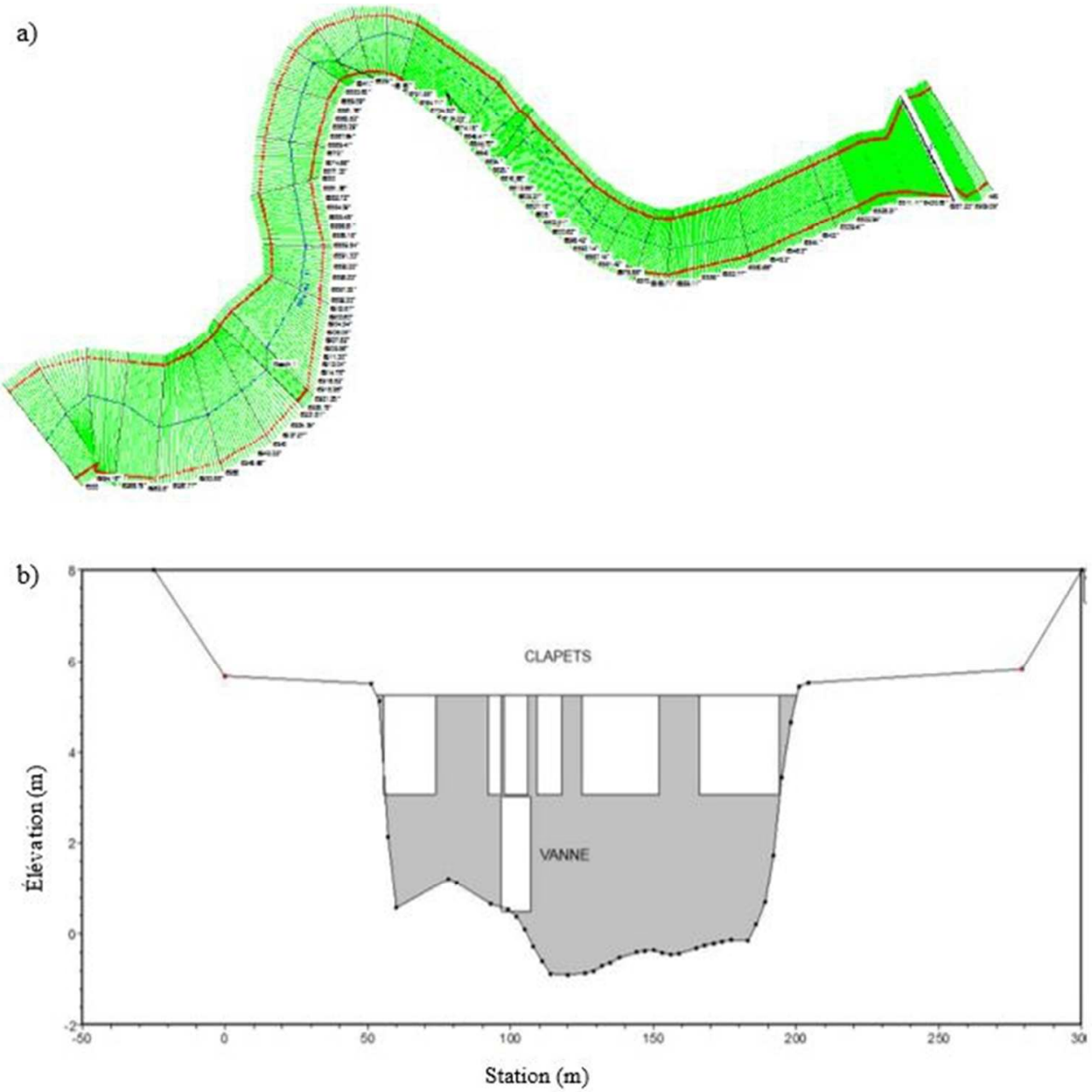

$173 \times 168 \mathrm{~mm}(96 \times 96 \mathrm{DPI})$ 

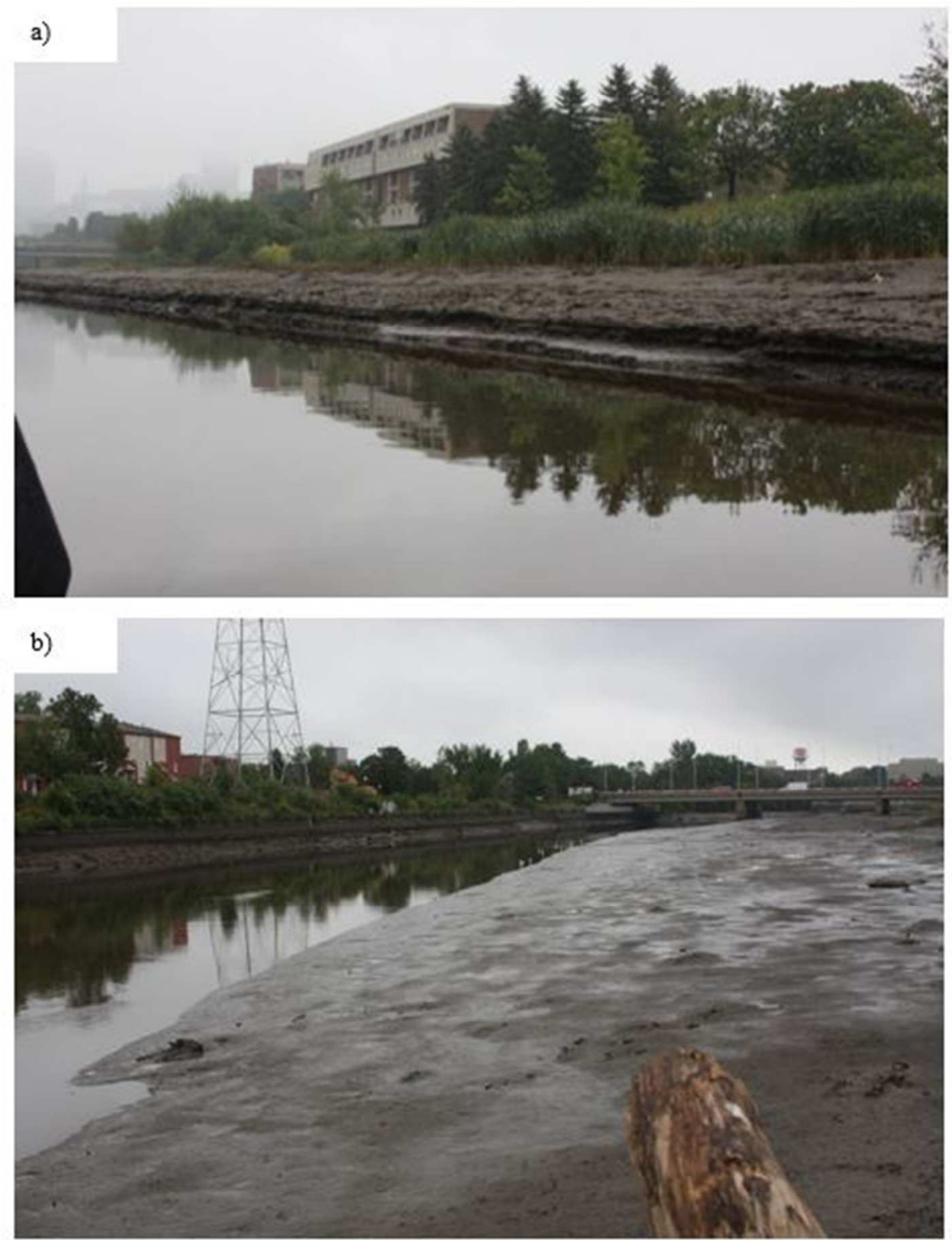

$128 \times 166 \mathrm{~mm}(96 \times 96 \mathrm{DPI})$ 


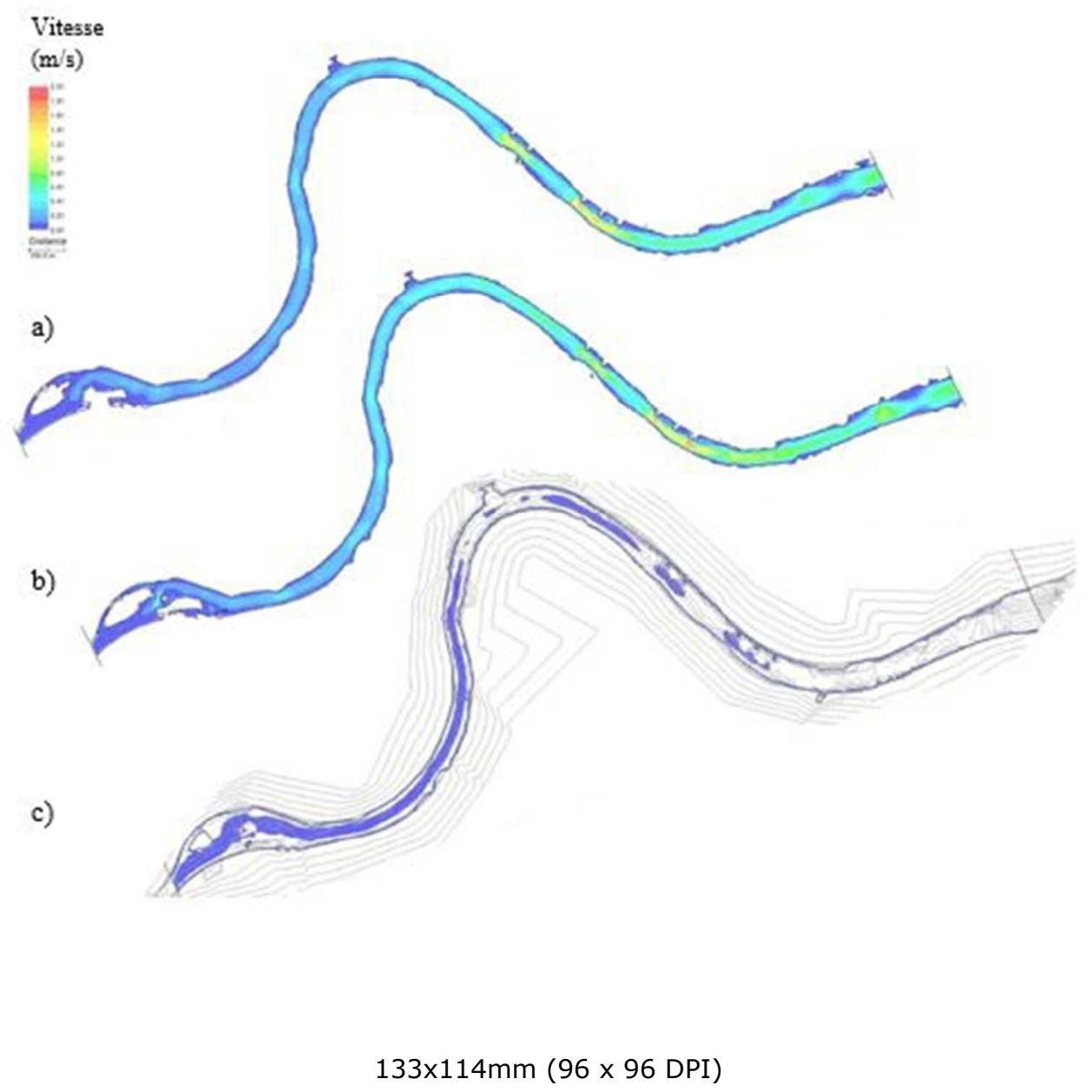

